\title{
Confinement-induced liquid ordering investigated by x-ray phase retrieval
}

\author{
Oliver Bunk, ${ }^{*}$ Ana Diaz, Franz Pfeiffer, Christian David, Celestino Padeste, Heilke Keymeulen, Philip R. Willmott, \\ Bruce D. Patterson, Bernd Schmitt, and Dillip K. Satapathy \\ Research Department Synchrotron Radiation and Nanotechnology, Paul Scherrer Institut, 5232 Villigen PSI, Switzerland \\ J. Friso van der Veen \\ Research Department Synchrotron Radiation and Nanotechnology, Paul Scherrer Institut, 5232 Villigen PSI, Switzerland \\ and ETH Zurich, 8093 Zürich, Switzerland. \\ Hua Guo and Gerard H. Wegdam \\ Van der Waals-Zeeman Instituut, Universiteit van Amsterdam, Valckenierstraat 65, 1018 XE Amsterdam, The Netherlands
}

(Received 29 August 2006; revised manuscript received 30 October 2006; published 6 February 2007)

\begin{abstract}
Using synchrotron x-ray diffraction, we have determined the ensemble-averaged density profile of colloidal fluids within confining channels of different widths. We observe an oscillatory ordering-disordering behavior of the colloidal particles as a function of the channel width, while the colloidal solution remains in the liquid state. This phenomenon has been suggested by surface force studies of hard-sphere fluids and also theoretically predicted, but here we see it by direct measurements of the structure for comparable systems.
\end{abstract}

DOI: 10.1103/PhysRevE.75.021501

PACS number(s): 61.10.Eq, 82.70.Dd, 61.20.-p, 68.15.+e

\section{INTRODUCTION}

Geometrical confinement can dramatically alter bulk properties of fluids like viscosity and density. This has important implications for fundamental processes such as lubrication, friction, crystallization, and transport through narrow pores. In a narrow, confined space, the fluid can change its phase behavior and may become even solidlike $[1,2]$. These phenomena are generally attributed to excluded volume effects, and become important when the confining dimensions are less than typically five to ten times the diameter of the fluid's constituents (molecules, micelles, or colloidal particles). Theoretical models predict for this limit a structural force acting on the confining surfaces that oscillates, in the case of hard-sphere liquids, with a period approximately equal to a particle diameter and, for charged colloids, with a larger period which reflects the range of the screened Coulomb force $[3,4]$. According to the contact value theorem [5], a positive (negative) force reflects an enhanced (lowered) local density near the confining walls. This suggests the presence of a layered (disordered) density next to the walls. Intuitively, the density oscillations induced by each of the two walls may interfere constructively or destructively, depending on whether an integer or a half-integer number of layers fits into the confining channel (Fig. 1).

Whereas oscillatory surface forces have been measured for many confined liquids [5,6], structural information is scarce, since the confinement geometry makes the fluid inaccessible to most structural probes. Optical microscopy has been employed to investigate the crystalline order of colloids following confinement-induced freezing [7], as well as the ordering of colloidal fluids against a single wall [8]. Confocal and standard video microscopy allow one to gain directly insight into the local dynamics of colloid solutions in the

\footnotetext{
*Electronic address: oliver.bunk@psi.ch
}

vicinity of a wall and has contributed significantly to the understanding of such systems $[9,10]$. As for any optical technique, the system has to be transparent, but contain contrast in the visible light regime between the colloidal particles and the surrounding fluid. The colloidal particles have to be sufficiently large and slow to allow detection and particles overlapping in the detected two-dimensional (2D) projection complicate data analysis [11]. Transmission electron microscopy is restricted to fluids with very low vapor pressures, while the generally unknown shape of the cantilever tip in atomic force microscopy restricts applications in this field. Determining the density profile of a fluid across the confining container with high resolution has so far remained

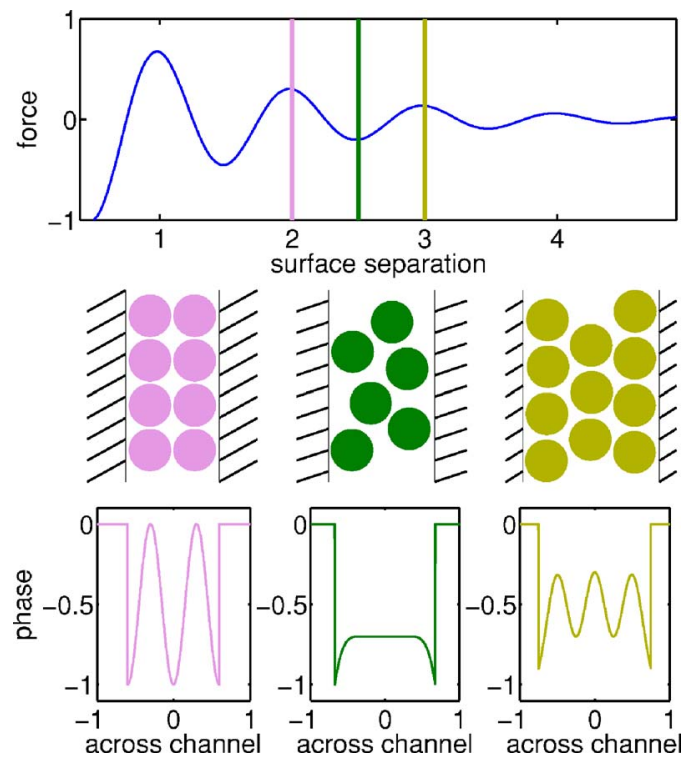

FIG. 1. (Color online) Oscillatory ordering. Schematic of the structural force as a function of surface separation (top panel), with the corresponding arrangement of the fluid particles (center panel) and density profile (bottom panel). 


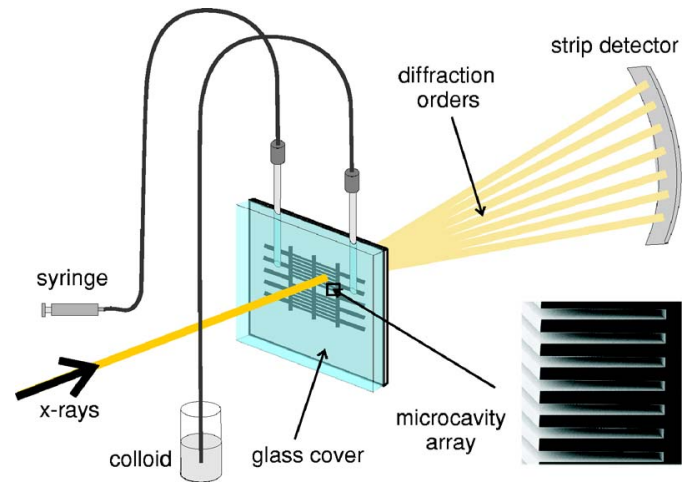

FIG. 2. (Color online) Experimental setup. The x-ray beam impinges under normal incidence onto the sample chip on which several different microfluidic arrays were prepared. The sample chip is covered with a glass plate to avoid evaporation. The diffracted intensity is recorded with a high-resolution microstrip detector. The inset shows a SEM image of a microfluidic array.

elusive, despite some promising proof-of-principle results using x-ray waveguiding [12] and x-ray reflectivity [13]. The microfluidic array phase profiling (MAPP) described here is complementary to most of the local probes listed above, since it provides ensemble-averaged information that is spatially resolved across the confining average channel rather than data about an individual channel.

\section{EXPERIMENT}

Instead of investigating single fluid containers, our approach was to use an array of microfluidic channels etched into a silicon chip for small-angle $\mathrm{x}$-ray scattering experiments. This approach has several advantages. By studying many identical containers at the same time, the ensemble average rather than a single entity is investigated, and the scattered x-ray signal is much stronger. The setup is insensitive to perturbations such as vibrations, since the dimensions of the fluid containers are not variable. Different dimensions of the confining channels are simply chosen by having a set of different arrays on the same sample, i.e., the same chip. The method is sensitive to electron density differences, i.e., no transparency or contrast of the components is needed in the visible light regime. Only the dimensions of the density fluctuations rather than the ordering constituents themselves have to be larger than the resolution and thereby for example ion concentrations can be measured.

We succeeded in producing such arrays with a high degree of perfection [14]. The data described in the present study were recorded for a chip with microfluidic arrays of $0.50,0.75,1.00$, and $1.25 \mu \mathrm{m}$ period and channel widths in the range of 280 to $615 \mathrm{~nm}$. The height of the structures is approximately $6 \mu \mathrm{m}$. Each array covers an area of $0.8 \times 0.8 \mathrm{~mm}^{2}$. A scanning electron microscopy (SEM) image of such a microfluidic array is shown in the inset of Fig. 2.

The microfluidic arrays were placed under normal incidence in the X-ray beam as schematically depicted in Fig. 2. The data were recorded at an x-ray energy of $11.804 \mathrm{keV}$, corresponding to a wavelength of $1.050 \AA$, at the Materials Science beamline of the Swiss Light Source, Paul Scherrer Institut [15]. A macroscopic beam of $0.5 \times 0.5 \mathrm{~mm}^{2}$ at the sample position and thereby most of the incident flux was used. The arrays act as diffraction gratings, and the intensities of the diffraction orders (Bragg peaks) for each grating were recorded with a high-resolution 1D microstrip detector [16] having $10 \mu \mathrm{rad}$ resolution corresponding to a minimum resolvable momentum transfer of $6 \times 10^{-4} \mathrm{~nm}^{-1}$. Combining three diffraction patterns obtained with different attenuation settings allowed us also to measure the low-order diffraction intensities, including the zeroth order, thereby eliminating the need for special treatment of missing low-frequency data $[17,18]$. The total exposure time for each microfluidic array was $135 \mathrm{~s}$. This time already includes a triple repeat of each exposure used to filter out spurious counting events by taking the median value of the recorded intensities.

Because the illumination is only partially coherent, the $\mathrm{x}$-ray data are sensitive to the average structure instead of individual channels and density profiles. In this sense, the current technique is more similar to crystallography than to lensless imaging using fully coherent illumination.

The colloidal solution consists of $10 \mathrm{vol} \%$ spheres of $109.2 \mathrm{~nm}$ average diameter in a refractive-index-matched solvent of $55 \%$ benzyl alcohol and $45 \%$ ethanol. The matching of the refractive index reduces van der Waals interactions. The polydispersity of the silica spheres is approximately $3 \%$. The concentration is well below the density for the formation of a crystalline layer at the wall or even capillary freezing [19]. In a second measurement in the same microfluidic channels, lithium chloride $(\mathrm{LiCl})$ in $0.4 M$ concentration was added. The additional $\mathrm{LiCl}$ ions in the salt solution shield the electrostatic potential of the charged colloid particles. The silica colloid particles then effectively become similar to a hard-sphere system.

\section{DATA ANALYSIS}

The iterative phase-retrieval algorithm presented here allows the determination of amplitude, phase, and thereby concentration profiles across the microfluidic channels in a model-independent way. For a model-dependent kinematic scattering approach to determine the concentration profile we refer to $[20,21]$.

\section{A. Coordinate system}

We are using a coordinate system in which $x$ is across the microfluidic channels, $y$ along the channels, and $z$ along the propagation direction of the $\mathrm{x}$ rays toward the detector. The diffraction measurements average over $y$ and take the ensemble average of many microfluidic channels. The origin $x=z=0$ is in the center of an average channel at the exit of the grating.

\section{B. Initial data treatment}

A periodic array of Gaussian peaks with the same width but individual intensities on an interpolated background was fitted to the spectrum for each grating in order to retrieve the 


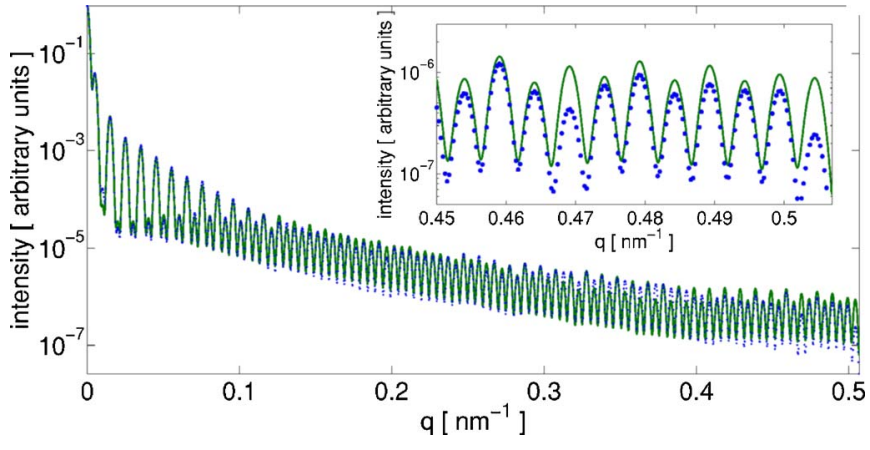

FIG. 3. (Color online) Experimental data. Retrieved (green line) and measured (blue dots) intensities on a logarithmic scale for 100 diffraction orders of a grating with $1.25 \mu \mathrm{m}$ period and $615 \mathrm{~nm}$ channel width.

integrated intensities of the array diffraction peaks with high precision. These integrated intensities contain the full information about the average electron density, spatially resolved across a microfluidic channel. See Fig. 3 for an example where the intensities are reconvoluted with the Gaussian peak to resemble the raw data.

\section{Phase retrieval}

Fourier transformation of the recorded intensities of the grating orders cannot yield density profiles because of the well-known phase problem, i.e., the loss of the x-ray phase information in the recorded data. To retrieve the phase information, iterative algorithms have proven to be very helpful.

For the present case of 1D density profiles from Bragg scattering data we attempted a combination of standard iterative phase-retrieval algorithms. We applied the following scheme. We start with a random solution and 15 iterations of Fienup's hybrid input-output algorithm [23], followed by 20 iterations of the Gerchberg-Saxton algorithm with the phaseselection option [24] repeated five times. We then apply three cycles of 15 iterations of the hybrid input-output algorithm, followed by 50 iterations of the Gerchberg-Saxton algorithm [22]. These are in total 370 iterations with the different algorithms. The method is computationally fast since we are dealing with a $1 \mathrm{D}$ data set.

The iterative phase retrieval was started for each data set, i.e., for each microfluidic array, 150 times with an initially random solution. For each of the refined 150 solutions, the similarity to all other solutions was calculated in the form of the mean squared deviation (MSD). The refined solutions are grouped into a large set of similar solutions and a small set of dissimilar ones. The level of rejection is the median value of the MSDs plus their standard deviation. Of the majority of similar solutions the average result is calculated. Typically fewer than $10 \%$ of the initially 150 solutions are rejected, i.e., for all the data presented here, well above 100 solutions are averaged.

The reconstructed field is complex valued and symmetric with respect to $q=0$. By including physical constraints in the algorithm, we succeeded in retrieving complex objects, i.e., both amplitude and phase, for a 1D problem in a unique way. Details about the MAPP technique including a detailed de-
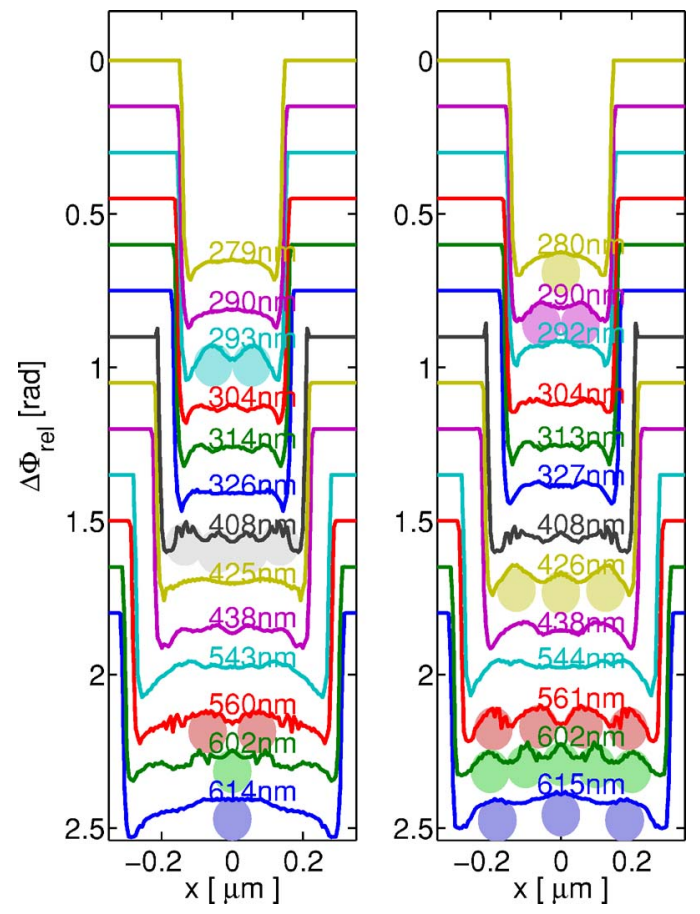

FIG. 4. (Color online) Phase profiles of charged silica colloids (left) and hard-sphere-like colloids (right) in the same microfluidic arrays. The profiles are offset vertically for clarity. The determined channel widths are shown for each profile. As we interpret oscillations as being caused by layering, i.e., increased concentration, we have highlighted these using spheres with the diameter of the colloid particles drawn to scale.

scription of the phase retrieval constraints can be found elsewhere [25]. For the discussion here, the most relevant aspect is that the precise position of the confining walls and thereby the width of the channels, the height of the channels and the phase profile of the fluid inside the channels are not constrained. This approach is therefore truly model independent: as much prior information as is available is used but none of the parameters to be determined is imposed in any way onto the system.

An example of the excellent agreement between measured and retrieved intensities is shown in Fig. 3. The data shown are reconvoluted with the Gaussian peak to resemble the original data before peak integration. The inset shows the deviations that occur for the highest orders due to the averaging of solutions [25].

Further evidence for the uniqueness and precision of the method is given by the retrieved phase profiles depicted in Fig. 4. In the left panel, phase profiles are shown for the charged silica colloid. The profiles in the right panel are for identical microfluidic arrays filled with the same colloid plus a $0.4 M \mathrm{LiCl}$ solution. The channel widths shown for each profile are determined as the full width at half average maximum of the phase profile. These values agree between both data sets for the same array to within $1 \mathrm{~nm}$, which is much better than the minimal single-pixel resolution of $6 \mathrm{~nm}$.

\section{Concentration profiles}

Propagation of $\mathrm{x}$ rays through the microfluidic array changes both phase and amplitude of the incident field. This 
is described by the real part $\delta$ and the imaginary part $\beta$ of the refractive index $n$ :

$$
n=1-\delta+i \beta .
$$

To convert the phase profiles into concentration profiles we use our knowledge about the refractive indices of the components of the system under investigation. In the example presented here the components are silicon, $\delta_{\mathrm{Si}}$ $=3.497 \times 10^{-6}$, silica colloidal particles, $\delta_{\text {silica }}=2.889 \times 10^{-6}$, and a refractive-index-matched solvent of 55\% benzyl alcohol and $45 \%$ ethanol, $\delta_{\text {solvent }}=1.521 \times 10^{-6}$ (all given for the x-ray wavelength of $\lambda=1.050 \AA$ ). The $\delta$ values of the colloidal solution relative to the confining silicon can be calculated from the volumetric content $c$ of the colloidal particles: $\delta(x)=c(x) \delta_{\text {silica }}+[1-c(x)] \delta_{\text {solvent }}-\delta_{\mathrm{Si}}$. Additionally we know the height of the microfluidic channels of $z_{c}=6 \mu \mathrm{m}$ by design and also from the measurements of the empty channels. The phase shift $\Delta \Phi$ caused by traversing the length $\Delta z$ of a medium with real part of the refractive index $\delta$ is given by $\Delta \Phi(x)=k \delta(x) \Delta z$. We can use this equation to convert the determined phase shift inside the gap $\Delta \Phi_{\text {rel }}$, relative to the surrounding silicon, into a concentration of colloidal particles $c_{\text {silica: }}$ :

$$
c_{\text {silica }}=\frac{\delta_{\mathrm{Si}}+\Delta \Phi_{\mathrm{rel}} / k z_{c}-\delta_{\text {solvent }}}{\delta_{\text {silica }}-\delta_{\text {solvent }}} .
$$

The concentration profiles shown in Fig. 5 are based on this conversion.

\section{RESULTS AND DISCUSSION}

The results reported here are for colloidal solutions in the fluid phase. At higher concentrations, colloids form ordered solid phases $[26,27]$. The phase profiles shown in the left panel of Fig. 4 are definitely far from being in the solid phase. Only weak modulations of the average density profile of the model fluid are visible. Dense crystalline ordering of the silica particles would lead to phase shifts much closer to the value of the surrounding silicon walls.

The most pronounced ordering effects are marked in Fig. 4 as circles, with the diameter of the colloidal particles of $109 \mathrm{~nm}$ drawn to scale. It is striking that only some of the channels exhibit ordering effects. For the solution with charged silica particles (left panel of Fig. 4) pronounced layerlike ordering is seen for the channels of $293 \mathrm{~nm}$ width whereas the $290 \mathrm{~nm}$ channels exhibit almost no ordering, and those with $304 \mathrm{~nm}$ probably show a mixture of several weakly ordered structures.

One may argue that, for the charged colloid, local charge differences are present and that the long-range forces lead to a variation in the ordering distance. The situation is better defined for colloids with salt solution. The ions in the salt solution shield the electrostatic potential, and the silica colloid particles effectively become a hard-sphere system: the paradigm of a model fluid.

For the hard-sphere fluid, pronounced layering in two, three, and four layers occurs at multiples of approximately $142 \mathrm{~nm}$ in channel width, i.e., at 290, 426, and $561 \mathrm{~nm}$ (see
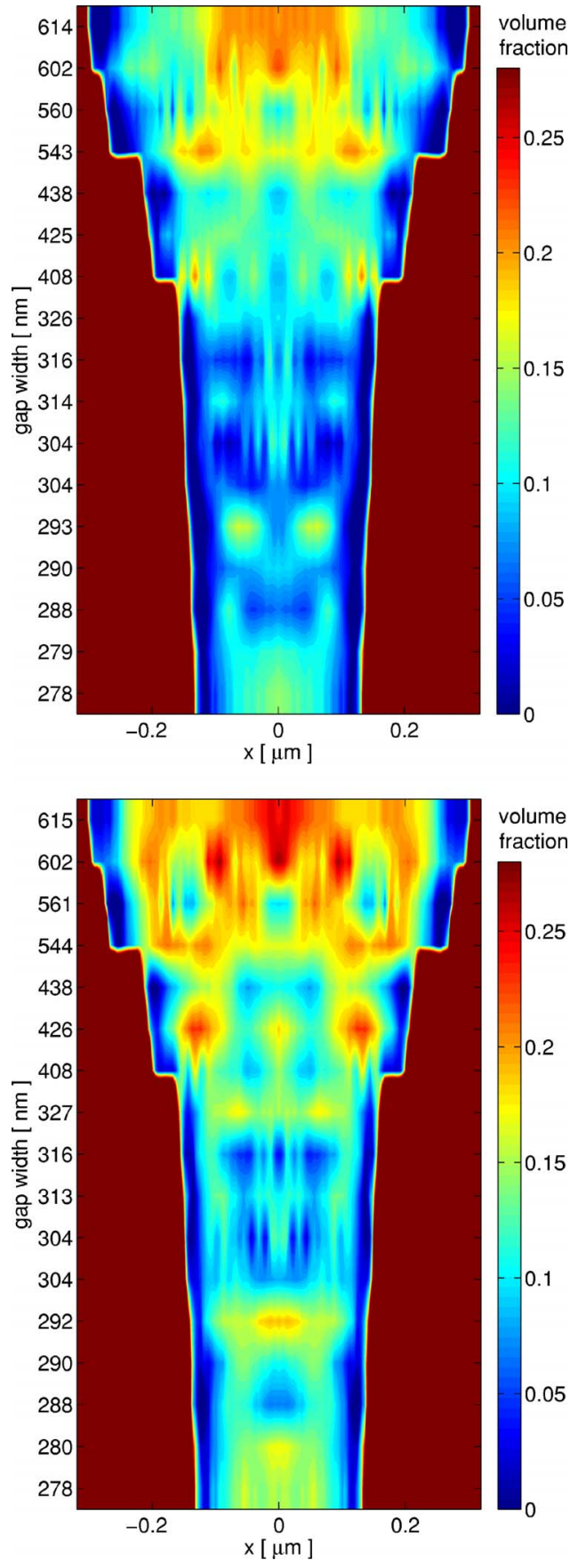

FIG. 5. (Color online) Volume fraction of colloidal particles. The silica concentration inside the microfluidic channels is shown as a contour plot for several channel widths for charged silica colloids (top) and hard sphere like colloids (bottom). The excluded volume effect of $0 \%$ volume fraction directly at the confining walls as well as pronounced increases due to the confinement are clearly visible. The dark red outer region is the area of the confining silicon walls.

right panel of Fig. 4). This is a clear fingerprint of confinement-induced oscillatory ordering with a typical distance larger than the diameter of the colloidal particles of $109 \mathrm{~nm}$. Pronounced ordering is observed only if the given channel width fits to the layering distance of the colloid inside. The data unequivocally show that the oscillatory struc- 
tural forces are directly linked to a sequence of layeringdisordering transitions, as illustrated in Fig. 1. We interpret this comparatively weak modulation of the density profile for a colloidal solution as a precursor state of the oscillatory crystalline ordering observed for dried systems created by electrodeposition [28], i.e., for the highest possible concentrations of colloid particles.

The concentration of the silica spheres calculated from the phase profiles in Fig. 4-spatially resolved across the confining channels - varies from $0 \%$ due to the excluded volume effect close to the walls to more than $25 \%$ in regions of high concentration (see Fig. 5). In addition, it is found that the average density across the channel width oscillates in phase with the degree of order (not shown). This is not unexpected, since the layering effect allows for a denser packing of the particles. A similar oscillation in the average density has been observed in a recent optical study of a confined molecular fluid [29].

At larger widths, the very regular layering breaks down, as seen for the $615 \mathrm{~nm}$ channels. The density fluctuations induced by the walls no longer overlap sufficiently to induce well-ordered layering, even though the density within the channels is still significantly increased compared to other arrays having only small signs of ordering effects.

Intermediate density profiles, which we interpret as superpositions of different states of local ordering, are also observed for many gap sizes where an integer number of layers does not fit into the confining gaps; see, for example, the channels with 327 and $438 \mathrm{~nm}$ width in the right panel of Fig. 4. This can be used to illustrate the complementarity of techniques: Local probes like video microscopy would find one or some of the realizations of the systems and this information can then be used to interpret the ensemble-averaged overall picture seen using the MAPP technique.

Gratings in photoresist layers have been used before to study the effects of confinement on the arrangement of colloidal particles and oscillatory packing densities have been found [28]. However, this study was performed on a completely different system, namely, densely packed colloidal crystals produced by electrodeposition, and a different, local probing technique was used: SEM on dried-out samples. This very elegant and straightforward approach was applicable since the focus was on colloid crystals for photonic applications rather than on dilute colloid solutions as in the present case.

\section{SUMMARY AND OUTLOOK}

We have identified oscillatory ordering of two, three and four layers in a silica hard-sphere model fluid confined in channels of submicrometer width. The colloidal solution nevertheless remains in the fluid state. A one-dimensional $\mathrm{x}$-ray phase-retrieval approach applied to $\mathrm{x}$-ray diffraction data from microfluidic arrays (MAPP) has enabled us to determine the oscillatory density profiles in a modelindependent way with a resolution in the $10 \mathrm{~nm}$ regime and to observe directly the structural oscillation underlying the well-known force oscillation.

The use of channel arrays for fluid confinement offers several advantages. Whereas a single channel typically contains only a picoliter of fluid, an array has approximately one thousand times more fluid. This allows a correspondingly lower irradiation dose per volume for the same scattered signal. Lowering the dose per volume is important for systems that are prone to radiation damage (e.g., hydrocarboncontaining fluids). Second, the wetting of arrays of channels with different dimensions by the same fluid enables accurate studies of confinement effects, free of systematic errors. Third, microfluidic arrays offer certain advantages for studies of dynamics using $\mathrm{x}$-ray photon correlation spectroscopy [30]. Scaling down the channels in these arrays to the range of $10-50 \mathrm{~nm}$ will offer unique opportunities for studies of confinement-induced ordering of proteins in solution. These kinds of system can be studied, since the MAPP technique is independent of the shape of the colloidal particles and, for example, rodlike particles or proteins are easily accessible.

The microfluidic arrays naturally lend themselves to studies of the flow of complex fluids in narrow channels, complementary to local studies of individual channels [31].

\section{ACKNOWLEDGMENTS}

This work was performed at the Swiss Light Source, Paul Scherrer Institut, Villigen, Switzerland. We thank Dominik Meister and Michael Lange for technical support and Harun H. Solak and C. Theiler for fruitful discussions.
[1] B. N. J. Persson, Comments Condens. Matter Phys. 17, 281 (1995).

[2] B. Bhushan, J. N. Israelachvili, and U. Landman, Nature (London) 374, 607 (1995).

[3] A. Trokhymchuk, D. Henderson, A. Nikolov, and D. T. Wasan, Langmuir 17, 4940 (2001).

[4] B. V. R. Tata, D. Boda, D. Henderson, A. Nikolov, and D. T. Watson, Phys. Rev. E 62, 3875 (2000).

[5] J. N. Israelachvili, Intermolecular and Surface Forces, 2nd ed. (Academic Press, New York, 1991).

[6] J. N. Israelachvili, Surf. Sci. Rep. 14, 109 (1992).

[7] P. Pieranski, L. Strzelecki, and B. Pansu, Phys. Rev. Lett. 50,
900 (1983).

[8] D. H. van Winkle and C. A. Murray, J. Chem. Phys. 89, 3885 (1988).

[9] C. A. Murray and D. G. Grier, Annu. Rev. Phys. Chem. 47, 421 (1996).

[10] J. C. Crocker and D. G. Grier, J. Colloid Interface Sci. 179, 298 (1996).

[11] J. Baumgartl and C. Bechinger, Europhys. Lett. 71, 487 (2005).

[12] M. J. Zwanenburg, J. F. Peters, J. H. H. Bongaerts, S. A. de Vries, D. L. Abernathy, and J. F. van der Veen, Phys. Rev. Lett. 82, 1696 (1999). 
[13] O. H. Seeck, H. Kim, D. R. Lee, D. Shu, I. D. Kaendler, J. K. Basu, and S. K. Sinha, Europhys. Lett. 60, 376 (2002).

[14] A. Diaz, C. David, H. Guo, H. Keymeulen, F. Pfeiffer, G. Wegdam, T. Weitkamp, and J. F. van der Veen, Physica B 357, 199 (2005).

[15] B. D. Patterson et al., Nucl. Instrum. Methods Phys. Res. A 540, 42 (2005).

[16] B. Schmitt, C. Brönnimann, E. F. Eikenberry, G. Hülsen, H. Toyokawa, R. Horisberger, F. Gozzo, B. Patterson, C. SchulzeBriese, and T. Tomizaki, Nucl. Instrum. Methods Phys. Res. A 518, 436 (2004).

[17] P. Thibault, V. Elser, C. Jacobsen, D. Shapiro, and D. Sayre, Acta Crystallogr., Sect. A: Found. Crystallogr. 62, 248 (2006).

[18] D. Shapiro et al., Proc. Natl. Acad. Sci. U.S.A. 102, 15343 (2005).

[19] M. Dijkstra, Phys. Rev. Lett. 93, 108303 (2004).

[20] A. Diaz and J. F. van der Veen, Thin Solid Films (to be published).

[21] A. Diaz, Ph.D. thesis No. 16851, ETH Zurich, 2006,
Available at: http://sls.web.psi.ch/view.php/beamlines/cs/ publications/thesis_Ana_Diaz.pdf

[22] R. W. Gerchberg and W. O. Saxton, Optik (Stuttgart) 35, 237 (1972).

[23] J. R. Fienup, Appl. Opt. 21, 2758 (1982).

[24] R. W. Gerchberg, Optik (Stuttgart) 74, 91 (1986).

[25] O. Bunk, A. Diaz, F. Pfeiffer, C. David, D. Satapathy, and J. F. van der Veen (unpublished).

[26] P. N. Pusey and W. van Megen, Nature (London) 320, 340 (1986).

[27] E. B. Sirota, H. D. Ou-Yang, S. K. Sinha, P. M. Chaikin, J. D. Axe, and Y. Fujii, Phys. Rev. Lett. 62, 1524 (1989).

[28] E. Kumacheva, R. K. Golding, M. Allard, and E. H. Sargent, Adv. Mater. (Weinheim, Ger.) 14, 221 (2002).

[29] M. Heuberger and M. Zäch, Langmuir 19, 1943 (2003).

[30] D. O. Riese, G. H. Wegdam, W. L. Vos, R. Sprik, D. Fenistein, J. H. H. Bongaerts, and G. Grübel, Phys. Rev. Lett. 85, 5460 (2000).

[31] L. Isa, R. Besseling, E. R. Weeks, and W. C. K. Poon, J. Phys.: Conf. Ser. 40, 124 (2006). 